\title{
Role of Src in angiopoietin 1-induced capillary morphogenesis of endothelial cells: Effect of chronic hypoxia on Src inhibition by PP2
}

\author{
Shigeru Kanda ${ }^{1,3}$, Hiroshi Kanetake ${ }^{2}$, and Yasuyoshi Miyata ${ }^{2}$
}

${ }^{1}$ Department of Molecular Microbiology and Immunology, Division of Endothelial Cell

Biology, and ${ }^{2}$ Department of Urology, Nagasaki University Graduate School of Biomedical

Science, 1-7-1 Sakamoto, Nagasaki 852-8501, Japan, and ${ }^{3}$ National Hospital Organization, Nagasaki Hospital, Sakuragi-machi 41-6, Nagasaki 850-8523, Japan.

Address for all correspondence:

Shigeru Kanda, M.D., Ph.D.,

National Hospital Organization

Nagasaki Hospital

Sakuragi-machi 41-6

Nagasaki 850-8523, Japan

Phone: +81 95-823-2261

Fax: +81 95-828--2616

E-mail: skanda-jua@umin.net 


\section{Abbreviations:}

Ang1, angiopoietin 1;

DMSO, dimethyl sulfoxide;

EBM, endothelial basal medium;

FBS, fetal bovine serum;

FGF, fibroblast growth factor;

HIF, hypoxia inducible factor;

HUVECs, human umbilical vein endothelial cells;

MDR, multidrug resistance.

MRP 1, multidrug resistance associated protein 1;

PlGF, placental growth factor;

siRNA, small interfering RNA;

TBS, Tris-buffered saline;

VEGF, vascular endothelial growth factor;

VEGFR, VEGF receptor;

Running title: Src activation in Ang1-induced capillary morphogenesis 


\section{Abstract}

Signal transduction pathways leading to angiopoietin 1 (Ang1)-induced capillary morphogenesis by endothelial cells remain poorly defined. Angiogenic cellular responses by endothelial cells may be modulated in vivo by chronic hypoxia, such as that induced by tumors. Here, we studied Ang1-induced capillary morphogenesis in human umbilical-vein endothelial cells (HUVECs) cultured chronically under normoxic (21\% oxygen) or hypoxic (1.5\% oxygen) conditions. Downregulation of Src using a small interfering RNA (siRNA) inhibited Ang1-induced capillary morphogenesis of HUVECs cultured under both conditions by blocking cell spreading and protrusion. Ang1 upregulated the Src-dependent secretion of vascular endothelial growth factor-A (VEGF-A). Blockade of endogenous VEGF-A also inhibited Ang1-induced capillary morphogenesis. Addition of exogenous VEGF-A restored cell spreading and protrusion, leading to Ang1-induced capillary morphogenesis of Src siRNA-treated HUVECs, suggesting that Ang1-induced VEGF-A secretion through Src was required for capillary morphogenesis. PP2 inhibited both Ang1-induced capillary morphogenesis and Src activation in HUVECs cultured under normoxic conditions, but the PP2 activity was significantly impaired in HUVECs cultured under hypoxic conditions. Expression of multidrug resistance-associated protein 1 (MRP 1) was upregulated in hypoxic HUVECs, and treatment with MRP 1 siRNA restored the inhibitory action of PP2. Taken together, our results suggest that Ang1 induces capillary morphogenesis in HUVECs through Src-dependent upregulation of endogenous VEGF-A. Conditions of chronic hypoxia impaired the effect of PP2, possibly via MRP 1.

Word count: 218

Key words: angiopoietin 1; capillary morphogenesis; Src; PP2; chronic hypoxia 


\section{Introduction}

Angiopoietins and their specific receptor tyrosine kinase, Tie 2, are implicated in the regulation of vascular and lymphatic vessel development and pathophysiological angiogenesis.

Angiopoietin 1 (Ang1) positively stimulates angiogenesis in certain tumors [1-4], inducing proliferation, migration and survival of endothelial cells [5-7]. Thus, Tie 2 signaling represents a potential target for the treatment of advanced cancers in human. Cellular responses in Ang1-treated endothelial cells are largely regulated through the Ras/mitogen-activated protein kinase and phosphoinositide 3-kinase/Akt pathways [5,6]. However, the role of Src family kinases in Ang1-induced cellular responses of endothelial cells has not yet been determined.

The Src family of protein tyrosine kinases plays a role in tumor progression and angiogenesis [8,9]. Src family kinases have been linked to angiogenic cellular responses of endothelial cells as well as in vivo angiogenesis promoted by fibroblast growth factor-2 (FGF-2), Ang2, vascular endothelial growth factor-A (VEGF-A), and hepatocyte growth factor [10-21]. Recently, anti-VEGF receptor-2 (VEGFR-2) blocking antibody inhibited angiogenesis and initial tumor growth in a mouse pancreatic islet tumor model. However, VEGFR-2 blockade alone failed to inhibit late-stage tumor growth, which was driven by FGF-2-induced angiogenesis [22]. This result suggested that blocking VEGF alone may induce VEGF-resistant angiogenesis and that the simultaneous inhibition of signal transduction pathways driven through multiple proangiogenic factor receptors may be required for complete inhibition of angiogenesis. Synthetic small-molecule protein kinase inhibitors, such as Src inhibitor PP2, may thus have the potential to block angiogenesis in advanced human cancers.

Many preclinical studies have used transplanted xenograft tumor models to assess the efficacy of antiangiogenic molecules. In these models in which tumor cells rapidly induce angiogenesis to evade host-tissue hypoxia, antiangiogenic therapies potently influence the 
tumor growth by inhibiting angiogenesis. However, in clinical trials of patients with malignant tumors, antiangiogenic monotherapy had little effect on tumor regression or on prolongation of patient survival. Human tumors grow slower than xenograft tumors, resulting in longer exposure of the endothelial cells to hypoxic conditions. The different characteristics of angiogenesis in transplanted tumors and autochthonous tumors may therefore reflect different sensitivities of the endothelial cells to synthetic protein kinase inhibitors as a function of oxygen tension. Assessments of these inhibitors with respect to angiogenesis have initially been conducted using cultured endothelial cells. Under normoxic conditions (usually 21\% oxygen in air), the oxygen tension of the culture medium is approximately $160 \mathrm{mmHg}$ [23]. Oxygen tension in normal human end-capillary blood is $45-50 \mathrm{mmHg}$, whereas that in hypoxic tumor tissue is $8-10 \mathrm{mmHg}$ [24]. Thus, it is possible that the sensitivity of endothelial cells to synthetic small-molecule protein kinase inhibitors may differ under normoxic and hypoxic (1.5-2.0\% $\left.\mathrm{O}_{2}\right)$ conditions.

The present study was designed to determine the role of Src in the signal transduction pathways leading to Ang1-induced capillary morphogenesis in both normoxia and hypoxia. The results showed that the sensitivity of Ang1-induced capillary morphogenesis to a Src family kinase inhibitor, PP2, was attenuated in endothelial cells cultured under chronic hypoxic conditions, possibly via upregulation of multidrug resistance-associated protein 1 (MRP 1). 


\section{Materials and methods}

\subsection{Materials}

Anti-Src polyclonal antibodies (N-16 for immunoprecipitation and SRC2 for immunoblotting), anti-Tie 2 antibody, anti-phosphotyrosine monoclonal antibody (PY-99), anti-MRP 1 antibody, non-targeting control small interfering RNAs (siRNA), and MRP 1 siRNA (sequences not available) were purchased from Santa Cruz Biotechnologies (Santa Cruz, CA). Histidine-tagged recombinant human Ang1, monoclonal anti-polyhistidine monoclonal antibody for cross-linking of histidine-tagged proteins, recombinant human VEGF-A, recombinant human placental growth factor 1 (PlGF-1), recombinant mouse VEGF-A, and the Quantikine ${ }^{\circledR}$ human VEGF-A ELISA kit were obtained from R \& D Systems (Minneapolis, MN). Growth factor-reduced Matrige ${ }^{\circledR}$ matrix and anti-hypoxia inducible factor- $1 \alpha(H I F-1 \alpha)$ antibody were purchased from BD Bioscience (Bedford, MA). Anti-pY ${ }^{418}$ Src antibody was obtained from BioSource International (Camarillo, CA), and anti-vinculin monoclonal antibody (hVIN-1) was from Sigma-Aldrich (St. Louis, MO). HiPerFect ${ }^{\circledR}$ transfection reagent and human Src siRNA (Hs_SRC_5_HP Validated siRNA, assessed by immunoblotting, sequence not available) were purchased from Qiagen (Tokyo, Japan). A pyrrolopyrimidine-derivative of Src inhibitor, PP2, was obtained from Merck (Tokyo); it was dissolved in dimethyl sulfoxide (DMSO) and stored at $-20^{\circ} \mathrm{C}$ until use. For experiments, the stock solution was further diluted with DMSO if required and dissolved in culture medium. The final concentration of DMSO in the culture medium was always $0.1 \%(\mathrm{~V} / \mathrm{V})$. Verapamil was obtained from Wako Pure Chemicals (Osaka, Japan), and was dissolved in water immediately before use. 


\subsection{Cell culture}

Human umbilical vein-derived endothelial cells (HUVECs) and the appropriate culture medium were obtained from Cambrex (Walkersville, MD). HUVECs were cultured in endothelial basal medium (EBM)-2 supplemented with 2\% fetal bovine serum (FBS), $20 \mathrm{ng} / \mathrm{ml}$ FGF-2, 20 ng/ml VEGF-A, 10 ng/ml epidermal growth factor, 20 ng/ml insulin-like growth factor-I, and $1 \mu \mathrm{g} / \mathrm{ml}$ hydrocortisone. Cells were cultured in an atmosphere of either a mixture of $95 \%$ air and $5 \% \mathrm{CO}_{2}$ (normoxic condition; $21 \% \mathrm{O}_{2}$ ), or $93.5 \% \mathrm{~N}_{2}, 5 \% \mathrm{CO}_{2}$, and $1.5 \% \mathrm{O}_{2}$ (hypoxic condition). $\mathrm{O}_{2}$ tension was monitored using an $\mathrm{O}_{2}$ Monitor, type MB1000 (CHINO Corporation, Tokyo). HUVECs were cultured under these conditions for at least 7 days before use. For the hypoxic culture, fresh culture medium was pre-incubated in the hypoxic atmosphere for overnight before use.

\subsection{Transfection of siRNA}

HUVECs were transfected with the indicated siRNA using HiPerFect ${ }^{\circledR}$ reagent. After 2 days, cells were detached from the wells and submitted to a capillary morphogenesis assay. Four thousand cells from each treatment were lysed and expression of Src and MRP 1 was examined by immunoblotting.

\subsection{Capillary morphogenesis assay}

The capillary morphogenesis assay was carried out as described previously [25]. In brief, HUVECs suspended in culture medium containing 0.5\% FBS were inoculated onto growth factor-reduced Matrigel ${ }^{\circledR}$, with or without treatment, and cultured for 24 h. To quantify 
the length of capillaries, three different phase-contrast photomicrographic images (x 4 objective) per well were taken, and the length of each capillary was measured using NIH image analysis software (version 1.64). Capillary length for cells without any treatment was set to 1.0. Values are expressed as means \pm SD of three images. To examine the kinetics of capillary morphogenesis, cells seeded onto Matrigel were serially photographed at indicated periods.

\subsection{Immunoblot analyses}

Two sets of cultures in 24-well plates were prepared; one set was used for immunoblotting and the other set for cell counts. Alternatively, siRNA-transfected cells were centrifuged and pelleted. Cells were lysed by boiled SDS-sample buffer, and the proteins from equal numbers of cells were separated on SDS-polyacrylamide gels. Proteins were electrotransferred onto polyvinylidene difluoride membranes. Membranes were incubated with the indicated antibodies, followed by incubation with peroxidase-conjugated secondary antibodies. Proteins were visualized by enhanced chemiluminescence reaction (Pierce Biotechnology, Rockford, IL) and exposure on X-ray film. To assess the tyrosine phosphorylation of Src and Tie 2, HUVECs cultured under either normoxic or hypoxic conditions were serum-starved for $2 \mathrm{~h}$ and then stimulated or left unstimulated with Ang1 for 8 min. Cells were washed with Tris-buffered saline, lysed in lysis buffer [7], and tyrosine phosphorylation of Src or Tie 2 was examined by immunoprecipitation followed by immunoblotting. Band intensities were quantified from scanned images of the blots using NIH Image (version 1.64). The relative ratio of the expressed amount of Src or MRP 1 was calculated as the ratio of Src or MRP 1 signal divided by the corresponding vinculin signal; each value was then normalized to the ratio obtained from control siRNA- transfected cells. 


\subsection{Measurement of secreted VEGF-A by HUVECs}

Two days after transfecion with either control or Src siRNA, HUVECs were suspended in medium containing $0.5 \%$ FBS, with or without $200 \mathrm{ng} / \mathrm{ml}$ Ang1, and seeded into 12-well plates. Twenty-four hours later, culture medium was collected and the concentration of VEGF-A was quantified using the Quantikine ${ }^{\circledR}$ human VEGF-A ELISA kit according to the protocol provided by the manufacturer, to a sensitivity of $15 \mathrm{pg} / \mathrm{ml}$ VEGF. Culture medium not incubated with HUVECs was used as a background value. The values are expressed as mean concentration \pm SD for triplicate wells.

\subsection{In vitro kinase assay}

In vitro kinase assay for Src was described previously [21]. In brief, cells were serum-starved for $2 \mathrm{~h}$ and were treated with either PP2 or 0.1\% DMSO. One hour later, cells were either stimulated or left unstimulated with Ang1 for 10 min. Src was immunoprecipitated and kinase assay was performed by the use of acid-denatured enolase as a substrate.

Incorporation of $\left[\gamma_{-}{ }^{32} \mathrm{P}\right]$ ATP into enolase was examined by SDS-PAGE, followed by autoradiography.

\subsection{Statistical analysis}

Values are presented as mean length \pm SD. Differences between two groups were analyzed using the Mann-Whitney’s U test. Differences were considered significant when the $P$ value was less than 0.05 . 


\section{Results}

3.1 Ang1 induces capillary morphogenesis by HUVECs cultured under both normoxic and hypoxic conditions

First, we showed that Ang1 was able to induce capillary morphogenesis of HUVECs cultured under normoxic or hypoxic conditions, in a dose-dependent manner (Fig. 1). The morphological changes were similar under both conditions of oxygen tension; cells became elongated and fused in the presence of Ang1.

\subsection{Src is involved in Ang1-induced capillary morphogenesis by HUVECs}

In light of the reported role of Src family kinases in tube formation or capillary morphogenesis of endothelial cells [11, 12, 14, 20, 21], we next examined the effect of expression of Src on Ang1-induced capillary morphogenesis by using siRNA technique. The Src siRNA at $10 \mathrm{nM}$ downregulated endogenous Src expression in HUVECs by 75\% compared to control siRNA-treated cells under both normoxic and hypoxic conditions, as assessed by densitometry (Fig. 2A). Fyn and Yes were not affected in the same experiments (data not shown). Downregulation of Src also potently inhibited Ang1-induced capillary morphogenesis by HUVECs cultured under both normoxic and hypoxic conditions (Fig. 2B), implicating Src in this process regardless of the ambient oxygen concentration. As shown in Fig. 2C, depletion of Src impaired capillary network formation by blocking normal cell spreading on a Matrigel surface and cell-cell contact via protrusions. 


\subsection{Src-dependent upregulation of VEGF-A is involved in Ang1-induced capillary morphogenesis}

We have previously shown that FGF-2-induced capillary morphogenesis by HUVECs requires endogenous VEGF-A-mediated signaling through VEGFR-1 [26]. In this report, the FGF-2-induced protrusion and extension of endothelial cells was inhibited in the presence of blocked VEGF-A signaling. In the fibroblast/endothelial cell co-culture model, VEGFR-2-mediated signaling via endogenous VEGF-A was required for Ang1-induced tube formation [27], and expression of VEGF-A was regulated through Src under normoxic and hypoxic conditions [28, 29]. These results suggest that endogenous VEGF-A may participate in Ang1-induced capillary morphogenesis. We therefore measured the secretion of VEGF-A by HUVECs cultured under both normoxic and hypoxic conditions. Cells transfected with either control or Src siRNA were cultured and culture medium was collected in the presence or absence of Ang1. Control siRNA- transfected cells showed significantly upregulated VEGF-A secretion following Ang1 treatment under both normoxic and hypoxic conditions, whereas Src siRNA transfection markedly downregulated the VEGF-A secretion by HUVECs (Fig. 3). This result indicated that Ang1 may stimulate the secretion of VEGF-A via Src family kinases. We next examined the effect of a blocking antibody against human VEGF-A, which inhibits the activity of human VEGF-A, but not of mouse VEGF-A [26], on Ang1-induced capillary morphogenesis. As shown in Fig. 4A, the VEGF-A blocking antibody significantly inhibited Ang1-induced capillary morphogenesis by HUVECs cultured under both normoxic and hypoxic conditions. Mouse VEGF-A (a ligand for both VEGFR-1 and -2), but not human PIGF-1 (a ligand only for VEGFR-1), restored this inhibition, suggesting that endogenous VEGF-mediated signaling through both VEGF-1 and VEGFR-2 was required for Ang1-induced capillary morphogenesis. We further examined the effect of exogenous 
VEGF-A on Ang1-induced capillary morphogenesis by Src-depleted HUVECs. As shown in Fig. 4B, exogenous VEGF-A restored Ang1-induced capillary morphogenesis in the Src-depleted HUVECs, but had no effect on the Ang1-induced capillary morphogenesis by HUVECs transfected with control siRNA. Exogenous VEGF-A also induced cell spreading on Matrigel and protrusion followed by network formation (Fig. 4C). These results suggest that Ang1-induced capillary morphogenesis requires Src-dependent secretion of endogenous VEGF-A.

3.4 Inhibition of Ang1-induced capillary morphogenesis in HUVECs by Src inhibitor PP2 is impaired under hypoxic culture conditions.

We next examined the effect of Src family kinase inhibitor PP2 on Ang1-induced capillary morphogenesis by HUVECs, and showed a significant inhibition under normoxic conditions (Fig. 5A). However, under hypoxic conditions, the sensitivity of Ang1-induced capillary morphogenesis to PP2 treatment was decreased. The effect of PP2 on Src activity in HUVECs was then examined. As shown in Figure 5B, Ang1 activated Src in HUVECs cultured under both normoxic and hypoxic conditions. However, PP2 did not efficiently decrease the Src activity in HUVECs cultured under hypoxic conditions, suggesting that the effect of PP2 is impaired in hypoxic cells. Immunoblot analysis by using phospho-specific antibodies against Src showed that PP2 decreased the pY418 of Src in HUVECs cultured under normoxic conditions, but not in HUVECs cultured under hypoxic conditions (Fig. 5C). We also showed that an upstream regulator of Src, Tie 2 receptor tyrosine kinase was efficiently autophosphorylated in HUVECs cultured under both normoxic and hypoxic conditions in the presence of Ang1 (Fig. 5D), suggesting that the activation of Tie 2 was not affected by chronic hypoxia. 
3.5 Impaired action of PP2 may be due to the upregulation of MRP 1

Under hypoxic conditions, the multidrug resistance (MDR 1) gene is upregulated through HIF-1 [30]. The impaired effect of PP2 under these conditions in HUVECs may therefore be due to an elevated expression of MDR 1 protein. To address this, we treated HUVECs with a broad MDR inhibitor, verapamil, and then examined the effect of PP2. Verapamil at $0.2 \mu \mathrm{M}$ significantly restored the inhibitory action of PP2 on Ang1-induced capillary morphogenesis and Src activation (data not shown). Since expression of MDR 1 protein was not altered by chronic hypoxia in HUVECs, we examined the expression of another transporter protein, MRP 1, the action of which is also inhibited by verapamil [31]. To compare the effect of acute and chronic hypoxia, some of the HUVECs cultured under normoxic conditions were transferred to hypoxic culture conditions for $8 \mathrm{~h}$ only. As shown in Fig. 6A, acute hypoxia markedly upregulated the HIF-1 $\alpha$ expression, which was extremely low in cells exposed to chronic hypoxia. Both acute and chronic hypoxic culture conditions strongly upregulated MRP 1 expression. These results indicate that upregulation of MRP 1 may affect the intracellular concentration of PP2 in HUVECs cultured under hypoxic condition. We next examined the effect of depleting MRP 1 protein on PP2-mediated inhibition of Ang1-induced capillary morphogenesis. HUVECs cultured under chronic hypoxia were transfected with either MRP 1 siRNA or control siRNA and cultured for 2 days. MRP 1 siRNA downregulated the expression of MRP 1 (Fig. 6B), and in these cells, Ang1-induced Src activation was inhibited by PP2 (Fig. 6C). Furthermore, the sensitivity of capillary morphogenesis by HUVECs to PP2 was restored by the MRP 1 depletion (Figure 6D), suggesting that MRP 1 is important for the sensitivity of HUVECs to PP2 under hypoxic conditions. 


\section{Discussion}

\subsection{Endogenous VEGF-A contributes to Ang1-induced capillary morphogenesis by HUVECs}

The signal transduction pathways that result in tube formation or capillary morphogenesis by Ang1-treated endothelial cells are poorly understood. The present study implicated Src as a likely downstream target of Tie 2, leading to capillary morphogenesis by endothelial cells. Downregulation of Src by siRNA inhibited cell spreading and protrusion of HUVECs on Matrigel, resulting in impaired capillary network formation, as well as decreasing the secretion of endogenous VEGF-A. Ang1-induced capillary morphogenesis was inhibited when endogenous VEGF-A was blocked. Addition of exogenous VEGF-A rescued Ang1-induced capillary morphogenesis by Src-depleted cells. Together, these results suggest that Ang1-induced secretion of VEGF-A through Src contributes significantly to capillary morphogenesis. Although a phosphoinositide 3-kinase (PI3-kinase) inhibitor, LY294002, modestly inhibit Ang1-induced capillary morphogenesis, but strongly inhibited the VEGF-A-induced capillary morphogenesis by HUVECs, PP2 weakly inhibited the capillary morphogenesis promoted by VEGF-A (S. Kanda, unpublished observation). In addition, VEGF-A had no further stimulatory effect on Ang1-induced capillary morphogenesis. Thus, Ang1-induced capillary morphogenesis may be partly attributed to VEGF-A-driven PI3-kinase activity. Depletion of Src expression impaired Ang1-induced cellular protrusion and extension of HUVECs, and similar defects were observed in PP2-treated cells (data not shown), suggesting that endogenous VEGF-A partially regulates cell shape and adhesion, which in turn requires turnover of focal adhesions and actin polymerization [32].

4.2 The effect of PP2 was impaired in HUVECs chronically exposed to hypoxia. 
Molecular targeting therapies in the future are expected to control advanced cancers in human. In particular, small-molecule protein kinase inhibitors have been tested widely clinical trials due to their easy accessibility (most are administered orally) and broad spectrum to a panel of protein kinases (targeting different kinds of kinases simultaneously). These reagents also inhibit both tumor progression and angiogenesis. In the present study, HUVECs cultured under hypoxic $\left(1.5 \% \mathrm{O}_{2}\right)$ conditions were used to simulate the tumor environment in human to assess the inhibitory activity of PP2. PP2 inhibited Ang1-induced capillary morphogenesis in normoxic HUVECs, but had little effect in cells cultured under chronic hypoxic conditions. The protein MDR 1 is upregulated by hypoxia [30], and we observed that verapamil restored the inhibitory action of PP2 on both capillary morphogenesis and Src activity of HUVECs cultured under hypoxic conditions, suggesting that multidrug resistance may be involved in the attenuated activity of PP2. Although MDR 1 expression was not upregulated under hypoxic conditions, another transporter MRP 1 was upregulated, and downregulation of MRP 1 in hypoxic HUVECs restored the sensitivity to PP2. These results therefore suggest that small molecule protein kinase inhibitors may be exported via the ATP-binding cassette (ABC) family of membrane ATPase transporters [33]. Interestingly, the action of a protein tyrosine kinase inhibitor of the 2-phenylaminopyrimidine series, imatinib, is impaired in imatinib-resistant leukemia cells [34] and is exported via MDR [35]. In addition, low expression of MRP 1 was a significant predictive factor of imatinib sensitivity in chronic myeloid leukemia [36]. Based on these findings and the results of this study, testing of small molecule protein kinase inhibitors in patients with advanced cancers should consider the activity of ABC membrane transporters.

\section{Acknowledgements}


We are grateful to Takumi Shimogama and members of the Nagasaki Radioisotope Center for their skilled and outstanding help. This work was supported by Grants-in-Aid from Japan Society for the Promotion of Science, (C)(2) 17591686 (S. Kanda) and (B) 17791080 (Y. Miyata). 


\section{References}

[1] W.S. Shim, M. Teh, A. Bapna, I. Kim, G.Y. Koh, P.O. Mack, R. Ge, Exp. Cell Res. 279 (2002) 299.

[2] J. Wang, K.C. Wu, D.X. Zhang, D.M. Fan, World J. Gastroenterol. 12 (2006) 2450.

[3] G. Zadeh, R. Reti, K. Koushan, Q. Baoping, P. Shannon, A. Guha, Neoplasia. 7 (2005) 1081.

[4] M.R. Machein, A. Knedla, R. Knoth, S. Wagner, E. Neuschl, K.H. Plate, Am. J. Pathol. 165 (2004) 1557.

[5] N. Jones, D.J. Dumont, Cancer Metastasis Rev. 19 (2000) 13.

[6] K.G. Peters, C.D. Kontos, P.C. Lin, A.L. Wong, P. Rao, L. Huang, M.W. Dewhirst, S. Sankar, Recent Prog. Horm. Res. 59 (2004) 51.

[7] S. Kanda, Y. Miyata, Y. Mochizuki, T. Matsuyama, H. Kanetake, Cancer Res. 65 (2005) 6820.

[8] R. Ishizawar, S.J. Parsons, Cancer Cell 6 (2004) 209.

[9] J.M. Summy, G.E. Gallick, Cancer Metastasis Rev. 22 (2003) 337.

[10] T. Shono, H. Kanetake, S. Kanda, Exp. Cell Res. 264 (2001) 275.

[11] P. Klint, S. Kanda, Y. Kloog, L. Claesson-Welsh, Oncogene 18 (1999) 3354.

[12] S. Tsuda, A. Ohtsuru, S. Yamashita, H. Kanetake, S. Kanda, Biochem. Biophys. Res. Commun. 290 (2001) 1354.

[13] W.W. Kilarski, N. Jura, P. Gerwins, Exp. Cell Res. 291 (2003) 70.

[14] Y. Mochizuki, T. Nakamura, H. Kanetake, S. Kanda, J. Cell Sci. 115 (2002) 175.

[15] B.P. Eliceiri, R. Paul, P.L. Schwartzberg, J.D. Hood, J. Leng, D.A. Cheresh, Mol. Cell 4 (1999) 915.

[16] A. Alavi, J.D. Hood, R. Frausto, D.G. Stupack, D.A. Cheresh, Science 301 (2003) 94. 
[17] H. He, V.J. Venema, X. Gu, R.C. Venema, M.B. Marrero, R.B. Caldwell, J. Biol. Chem. 274 (1999) 25130.

[18] R. Abu-Ghazaleh, J. Kabir, H. Jia, M. Lobo, I. Zachary, Biochem. J. 360 (2001) 255.

[19] M. McMullen, R. Keller, M. Sussman, K. Pumiglia, Oncogene 23 (2004) 1275.

[20] X.Q. Werdich, J.S. Penn, Angiogenesis 9 (2006) 1.

[21] S. Kanda, H. Kanetake, Y. Miyata Y, Biochem. Biophys. Res. Commun. 344 (2006) 617.

[22] O. Casanovas, D.J. Hicklin, G. Bergers, D. Hanahan, Cancer Cell 8 (2005) 299.

[23] M.V. Blagosklonny, Cancer Cell 5 (2004) 13.

[24] M. Hockel, P. Vaupel, J. Natl. Cancer Inst. 993 (2001) 266.

[25] S. Kanda, Y. Mochizuki, T. Suematsu, Y. Miyata, K. Nomata, H. Kanetake, J. Biol. Chem. 278 (2003) 8244.

[26] S. Kanda, Y. Miyata, H. Kanetake, J. Biol. Chem. 279 (2004) 4007.

[27] M. Saito, M. Hamasaki, M. Shibuya, Cancer Sci. 94 (2003) 782.

[28] D. Mukhopadhyay, L. Tsiokas, X.M. Zhou, D. Foster, J.S. Brugge, V.P. Sukhatme, Nature 375 (1995) 577.

[29] L.M. Ellis, C.A. Staley, W. Liu, R.Y. Fleming, N.U. Parikh, C.D. Bucana, G.E. Gallick, J. Biol. Chem. 273 (1998) 1052.

[30] K.M. Comerford, T.J. Wallace, J. Karhausen, N.A. Louis, M.C. Montalto, S.P. Colgan, Cancer Res. 62 (2002) 3387.

[31] A. Boumendjel, H. Baubichon-Cortay, D. Trompier, T. Perrotton, A. Di Pietro, Med Res Rev. 25 (2005) 453.

[32] S. Kanda, Y. Miyata, H. Kanetake, Cell Signal. 16 (2004) 1273.

[33] C. Özvegy-Laczka, J. Cserepes, N.B. Elkind, B. Sarkadi, Drug Resist. Updat. 8 (2005) 15. 
[34] F.X. Mahon, F. Belloc, V. Lagarde, C. Chollet, F. Moreau-Gaudry, J. Reiffers, J.M. Goldman, J.V. Melo, Blood 101 (2003) 2368.

[35] N. Widmer, S. Colombo, T. Buclin, L.A. Decosterd, Blood 102 (2003) 1142.

[36] T. Lange, C. Gunther, T. Kohler, R. Krahl, S. Musiol, S. Leiblein , H.K. Al-Ali, I. van Hoomissen, D. Niederwieser, M.W. Deininger, Blood 101 (2003). 2152. 


\section{Figure legends}

Fig. 1. Ang1 induces capillary morphogenesis by HUVECs cultured under both normoxic and hypoxic conditions in a dose-dependent manner. HUVECs cultured under normoxic or hypoxic (1.5\% oxygen in $\mathrm{N}_{2}$ ) for more than 7 days were harvested, suspended in culture medium containing $0.5 \%$ FBS, and cultured onto growth factor-reduced Matrigel in the presence or absence of Ang1 at indicated concentrations for $24 \mathrm{~h}$. Photographs were taken under a phase-contrast microscope. Capillary length of cells without any treatment was set to 1.0. Values are expressed as means \pm SD of three images. Bar, $20 \mu \mathrm{m}$. Reproducible results were obtained from two independent experiments.

Fig. 2. Downregulation of Src inhibits Ang1-induced capillary morphogenesis by HUVECs cultured under both normoxic and hypoxic conditions. (A) Src siRNA at $10 \mathrm{nM}$ efficiently downregulated Src in HUVECs cultured under both normoxic and hypoxic conditions. HUVECs were transfected with either control siRNA or Src siRNA at $10 \mathrm{nM}$ and cultured for 48 h. Cells were harvested and equal number of cells were lysed, run on SDS-PAGE, and Src expression was examined by immunoblotting. Vinculin expression was measured as a loading control. The remaining cells were used for the capillary morphogenesis assay as described below. (B) Downregulation of Src by siRNA at $10 \mathrm{nM}$ inhibited Ang1-induced capillary morphogenesis by HUVECs cultured under both normoxic and hypoxic conditions. Cells transfected with indicated siRNA were suspended in EBM-2 containing 0.5\% FBS and seeded onto Matrigel. Ang1 (200 ng/ml) was added to the indicated cells. Capillary morphogenesis was estimated as described in the legend of Fig. 1. Values are expressed as means \pm SD of three images. Bar, $100 \mu \mathrm{m}$. Reproducible results were obtained from two independent experiments. (C) Downregulation of Src by siRNA at $10 \mathrm{nM}$ inhibited Ang1-induced cell spreading on 
Matrigel and protrusion. Cells transfected with either control siRNA or Src siRNA were seeded onto a Matrigel surface. Micrographs were taken consecutively at indicated periods.

Fig. 3. Effect of Src siRNA on VEGF-A secretion by HUVECs cultured under normoxic and hypoxic conditions. HUVECs treated with the indicated siRNA at $10 \mathrm{nM}$ were cultured for 2 days under either normoxic or hypoxic conditions and then cells were harvested and seeded onto 24-well plates. Cells in EBM-2 medium containing 0.5\% FBS supplemented with or without Ang1 (200 ng/ml) were cultured for $24 \mathrm{~h}$ and conditioned medium was collected. VEGF-A concentration was measured by Quantikine ELISA kit. VEGF-A content was normalized to cell number. Values are expressed as means \pm SD of triplicate wells. Similar results were obtained from two independent experiments.

Fig. 4. (A) Blockage of endogenous VEGF-A by blocking antibody inhibits Ang1-induced capillary morphogenesis by HUVECs under both normoxic and hypoxic conditions. HUVECs were suspended in EBM-2 containing 0.5\% FBS and seeded onto Matrigel. Normal goat IgG or anti-VEGF-A blocking antibody at a concentration of $2.5 \mu \mathrm{g} / \mathrm{ml}$, Ang1 (200 ng/ml), mouse VEGF-A (20 ng/ml), and human PlGF-1 (50 ng/ml) was added to the cells as indicated. Capillary morphogenesis was estimated as described in the legend of Figure 1. Values are expressed as means \pm SD of three images. Bar, $100 \mu \mathrm{m}$. Reproducible results were obtained from two independent experiments. (B) Addition of exogenous VEGF-A restored Ang1-induced capillary morphogenesis by HUVECs transfected with Src siRNA (10 nM). HUVECs transfected with the indicated siRNA were cultured for 2 days under either normoxic or hypoxic conditions and then cells were harvested and seeded onto Matrigel and cultured in the presence or absence of indicated samples. Values are expressed as means \pm SD of three images. Reproducible results were obtained from two independent experiments. (C) Addition 
of exogenous VEGF-A restored Ang1-induced cell spreading on Matrigel and protrusion by HUVECs transfected with Src siRNA (10 nM). Cells transfected with Src siRNA were seeded onto the surface of Matrigel and cultured in the presence of indicated samples. Micrographs were taken consecutively at indicated periods. Reproducible results were obtained from two independent experiments.

Fig. 5. PP2 inhibits Ang1-induced capillary morphogenesis of HUVECs cultured under normoxic conditions, but not under hypoxic conditions. (A) HUVECs were suspended in EBM-2 containing $0.5 \%$ FBS and seeded onto Matrigel. Indicated samples were added to the cells and culture was continued for $24 \mathrm{~h}$. Values are expressed as means \pm SD of three images. Bar, $100 \mu \mathrm{m}$. Reproducible results were obtained from three independent experiments. (B) PP2 failed to inhibit Ang1-induced Src activation in HUVECs cultured under hypoxic condition. HUVECs were cultured in $6 \mathrm{~cm}$ dishes and incubated with serum-free medium with or without PP2 $(1 \mu \mathrm{M})$ for $2 \mathrm{~h}$, then either stimulated or left unstimulated with $200 \mathrm{ng} / \mathrm{ml}$ of Ang1 for 10 min. Src was immunoprecipitated from the $90 \%$ of the cell lysate and in vitro kinase assay was performed. The remaining $10 \%$ of each lysate was assessed for the loaded amount of protein by immunoblotting. Reproducible results were obtained from two independent experiments. (C) PP2 failed to inhibit Ang1-induced autophosphorylation of Src in HUVECs cultured under hypoxic condition. HUVECs were cultured in 6-cm dishes and incubated with serum-free medium with or without PP2 $(1 \mu \mathrm{M})$ for $2 \mathrm{~h}$, then either stimulated or left unstimulated with $200 \mathrm{ng} / \mathrm{ml}$ of Ang1 for $10 \mathrm{~min}$. Src was immunoprecipitated and site-specific phosphorylation was examined by immunoblotting. Reproducible results were obtained from two independent experiments. (D) Tyrosine phosphorylation of Tie 2 was not affected by chronic hypoxia. HUVECs grown in 10-cm dishes were serum-starved for $2 \mathrm{~h}$ and were either stimulated or left 
unstimulated with $200 \mathrm{ng} / \mathrm{ml}$ of Ang1 for $10 \mathrm{~min}$. Tie 2 was immunoprecipitated and autophosphorylation was examined by immunoblotting.

Fig. 6. Impaired activity of PP2 in HUVECs cultured under hypoxic condition may be due to an increase in MRP 1 protein. (A) Expression of MRP 1 was upregulated in HUVECs cultured under hypoxic conditions. Cells cultured in 24-well plates were lysed and equal cell numbers were resolved by SDS-PAGE, followed by immunoblotting. Expression of vinculin was examined as a loading control. Reproducible results were obtained from two independent experiments. (B) Downregulation of MRP 1 by siRNA. HUVECs cultured under hypoxic conditions were transfected with either control siRNA or MRP 1 siRNA at $50 \mathrm{nM}$ and two days later, cells were harvested, counted and proteins from 4 x $10^{4}$ cells were separated by SDS-PAGE, followed by immunoblotting. (C) Downregulation of MRP 1 restored the inhibitory action of PP2 on Ang1-induced Src activation. Hypoxic HUVECs transfected with either control siRNA or MRP 1 siRNA at 50 nM were either treated or left untreated with Ang1 at $200 \mathrm{ng} / \mathrm{ml}$ for $8 \mathrm{~min}$ in the presence or absence of PP2 as described in the legend of Fig. $5 \mathrm{~B}$. Src was immunoprecipitated followed by immunoblotting to examine the activation of Src. (D) The remaining HUVECs prepared in (B) were examined for Ang1-induced capillary morphogenesis in the presence or absence of PP2. Values are expressed as means \pm SD of three images. Bar, $100 \mu \mathrm{m}$. Reproducible results were obtained from two independent experiments. 

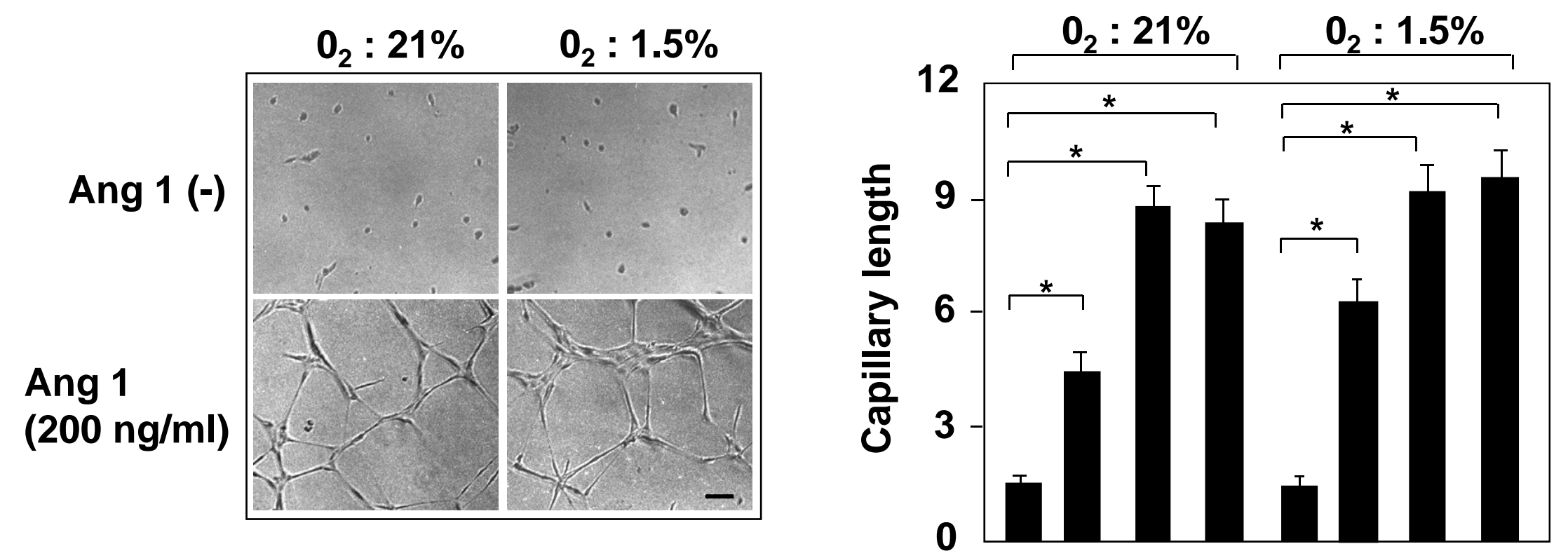

Ang 1: $0 \quad 100200400 \quad 0 \quad 100200400$ (ng/ml)

Fig. 1. S. Kanda et al. 
A

$$
\mathrm{O}_{2}: 21 \% \quad \mathrm{O}_{2}: 1.5 \%
$$

Cont. siRNA (nM) : $\begin{array}{lllllll}10 & 0 & 0 & 10 & 0 & 0\end{array}$

$\begin{array}{lllllll}\text { Src siRNA (nM) : } & 0 & 1 & 10 & 0 & 1 & 10\end{array}$

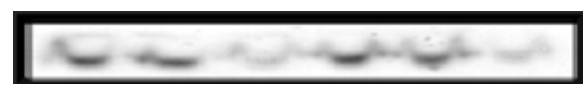

Src/vinculin ratio : $\begin{array}{llllll}1.00 & 0.95 & 0.24 & 1.00 & 0.87 & 0.21\end{array}$ IB : anti-Src

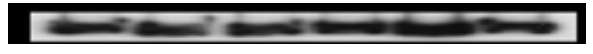

IB : anti-vinculin

B

$\begin{array}{lll}0_{2}: 21 \% & 0_{2}: 1.5 \%\end{array}$
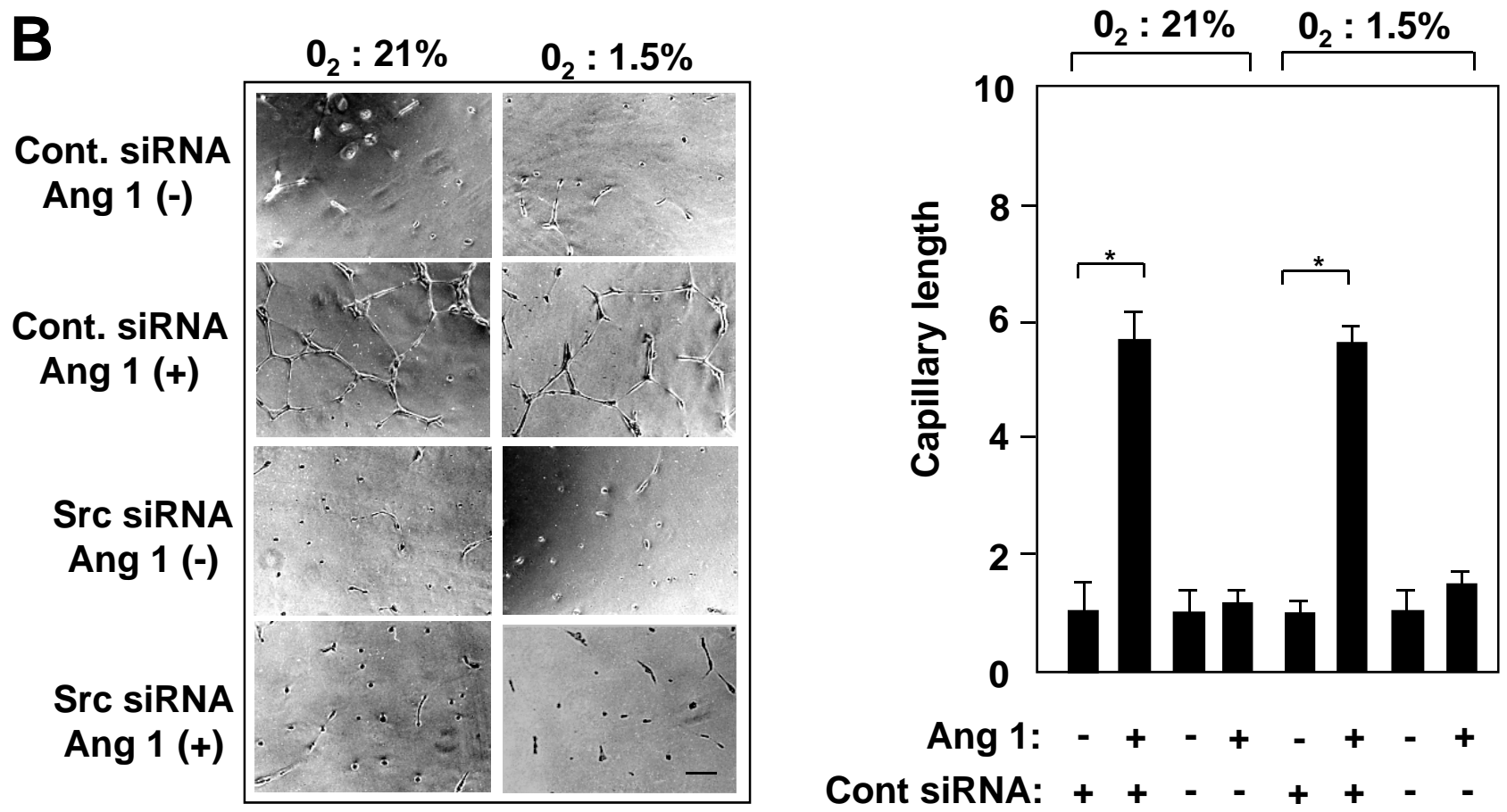

Ang 1: $-++\quad+\quad+\quad+$

Cont siRNA: $++-{ }_{-}++--$

Fig. 2. S. Kanda et al. 
C

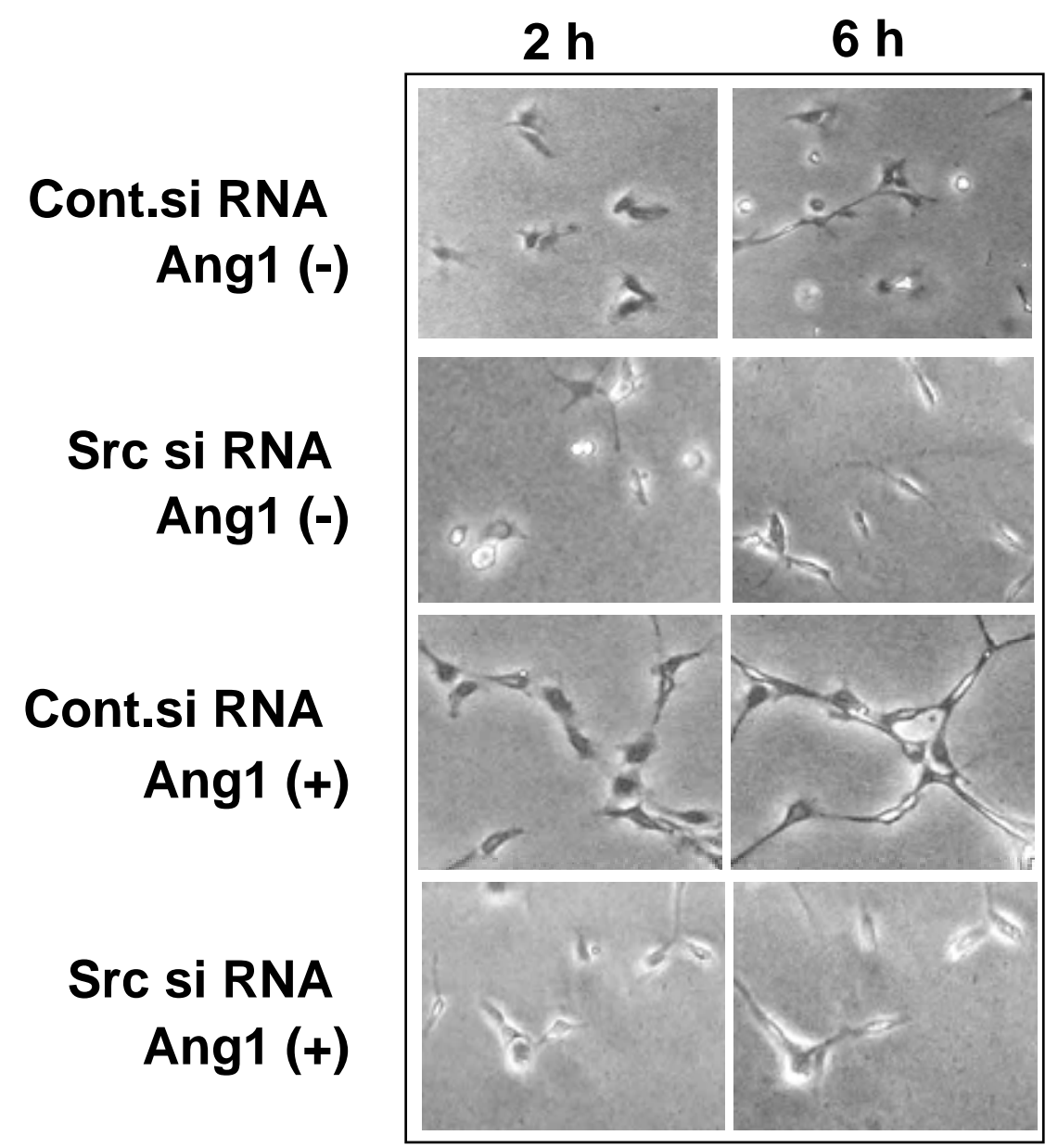

Fig. 2. S. Kanda et al. 


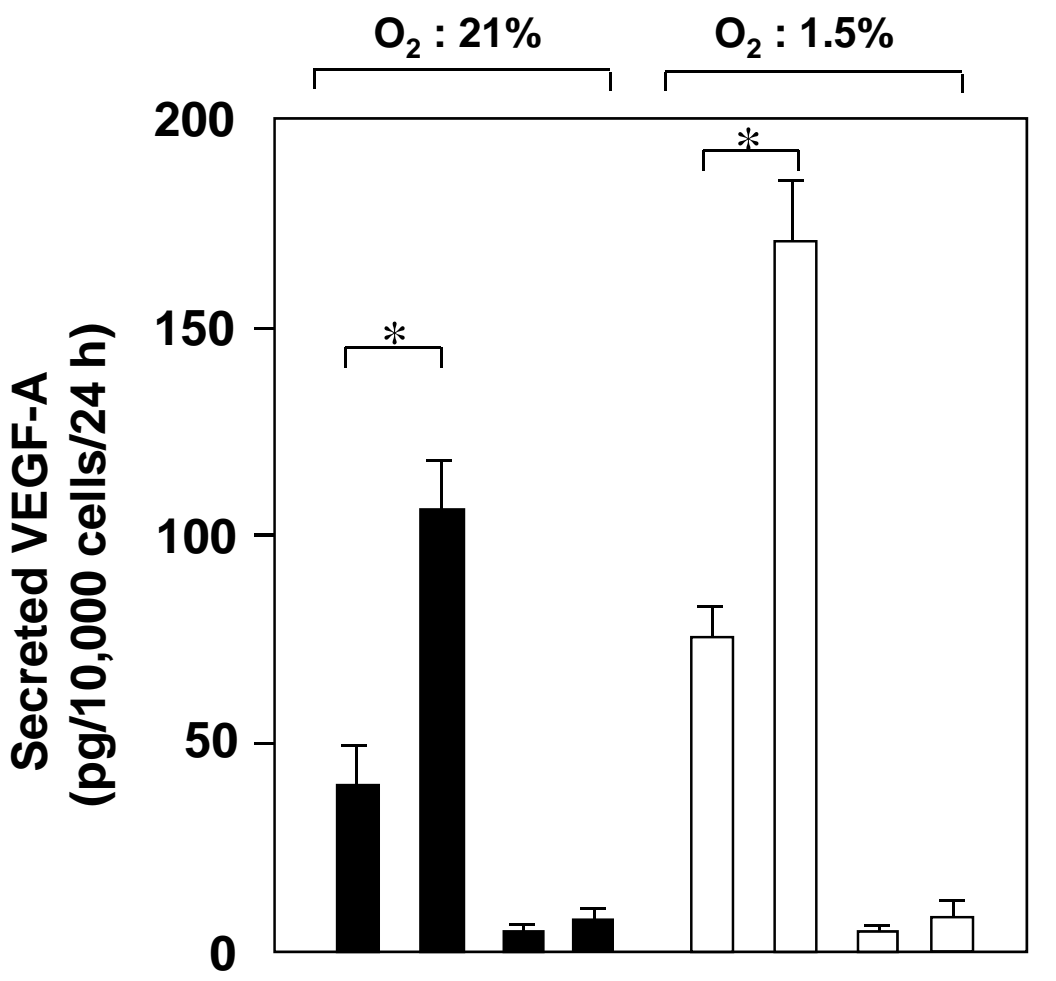

Control siRNA :

Src siRNA : $\quad-\quad+\quad+\quad-\quad++$

Ang1 : $-+{ }_{-}+{ }_{-}+-+$

Fig. 3. S. Kanda et al. 
A

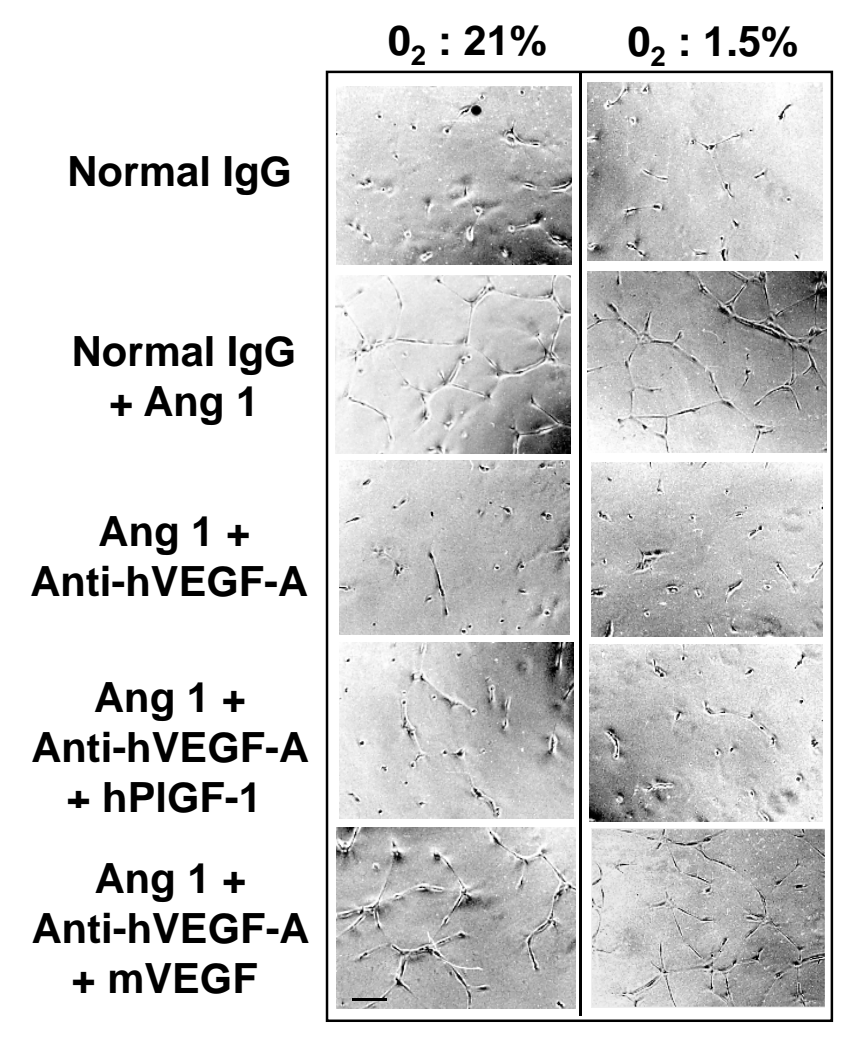

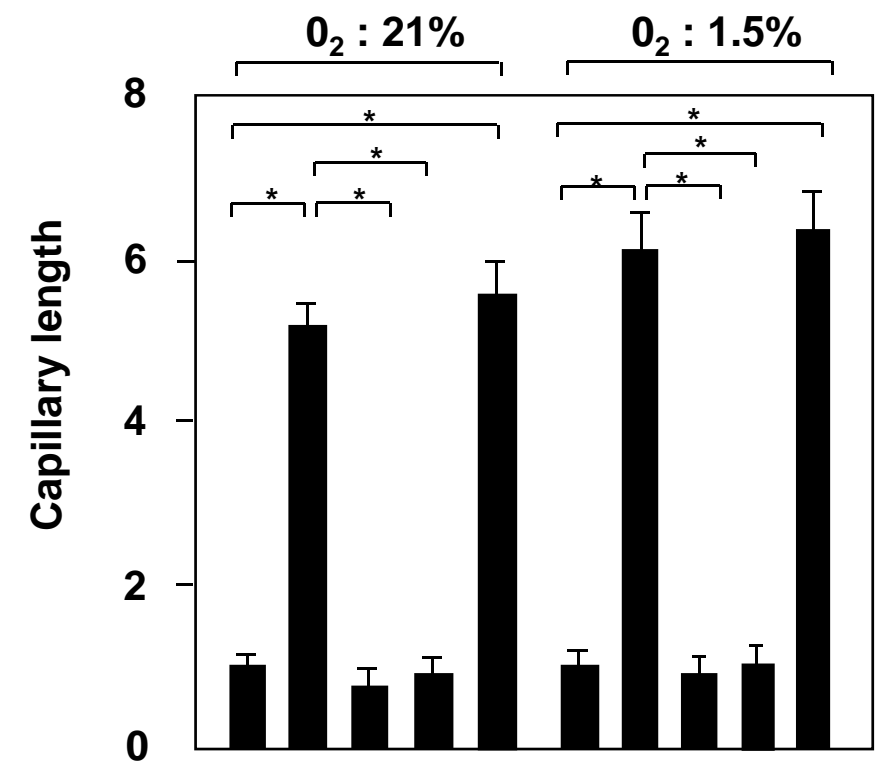

Ang 1: $-+++++++{ }_{+}^{+}$

Normal IgG : ++---++--

Anti-hVEGF-A: - $-+++-\quad+++$

hPIGF-1: - - $\quad+\quad-\quad-\quad-+$

mVEGF-A: - $\quad-\quad-\quad-\quad+\quad-\quad-\quad-\quad+$

Fig. 4. S. Kanda et al. 
B

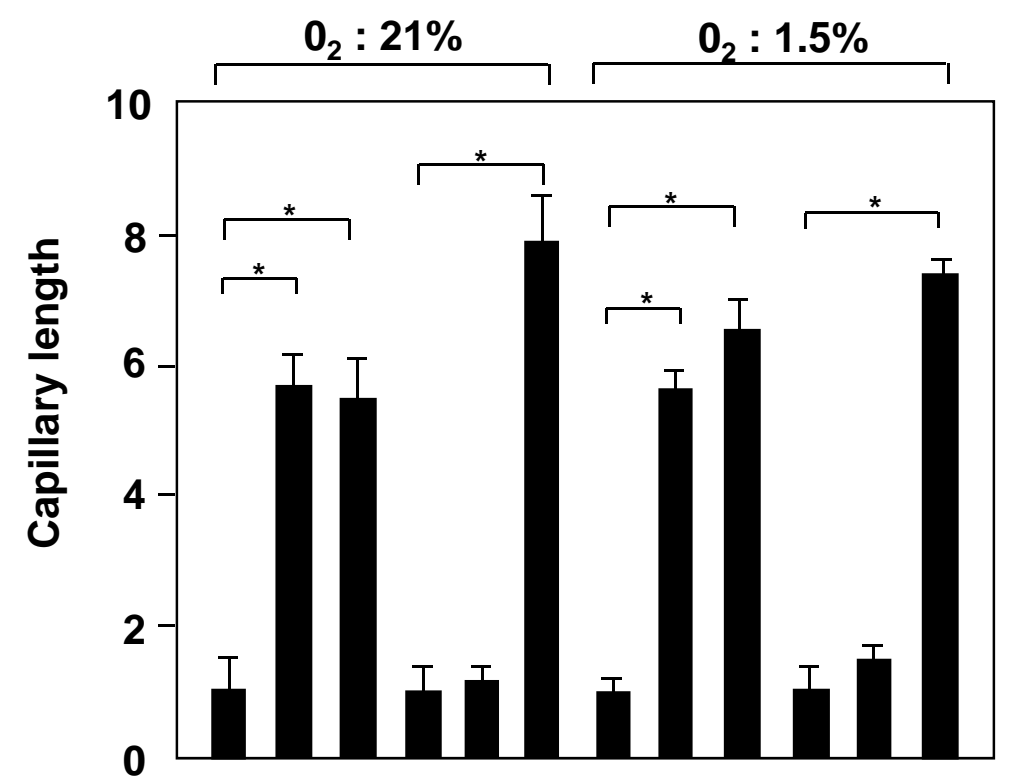

Ang 1:

VEGF-A:

Control siRNA:

Src siRNA:
C

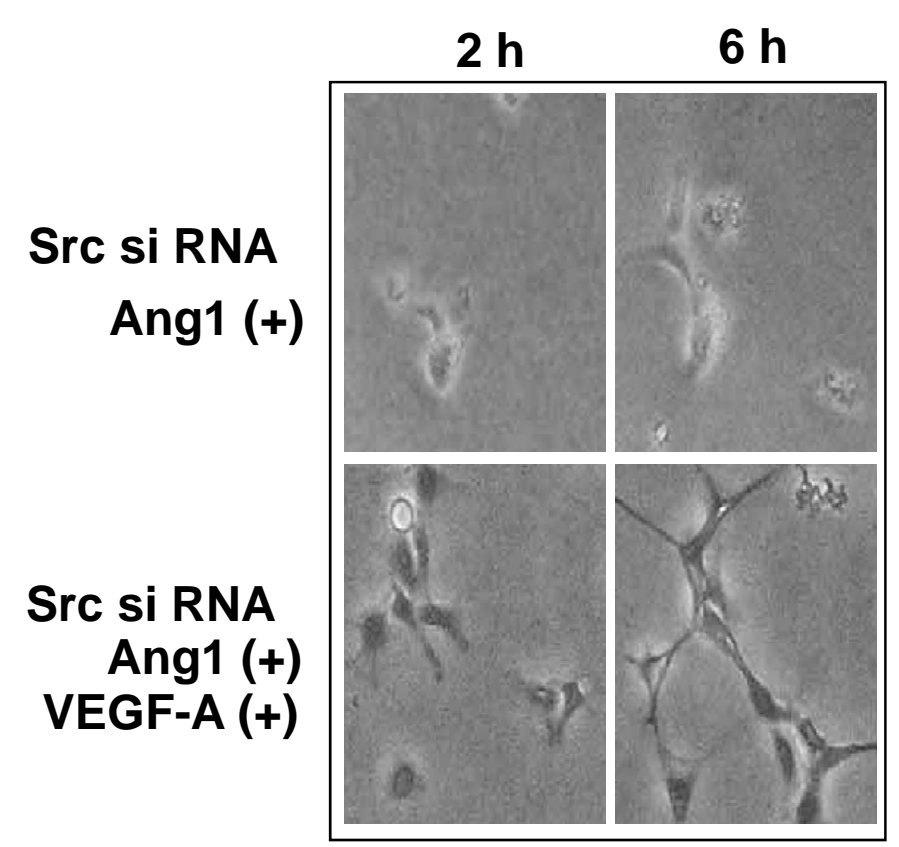

Fig. 4. S. Kanda et al. 

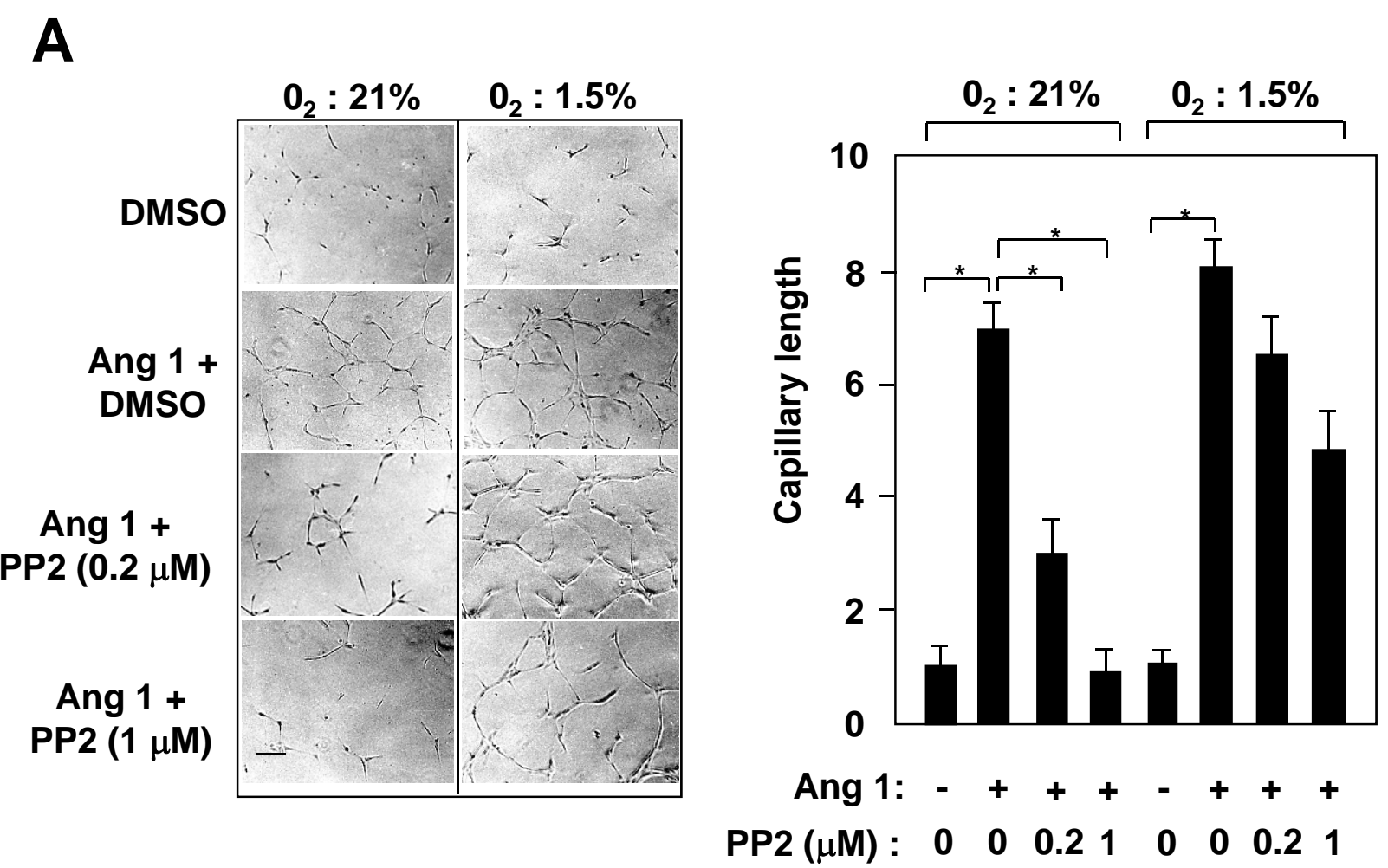

Fig 5. S. Kanda et al. 


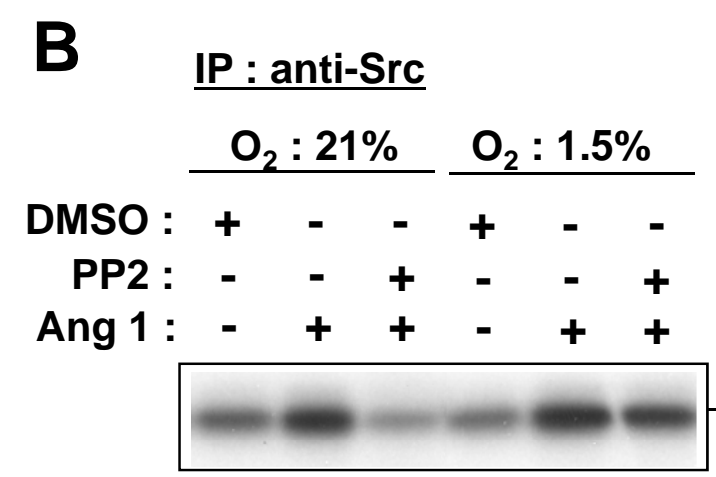

In vitro kinase assay

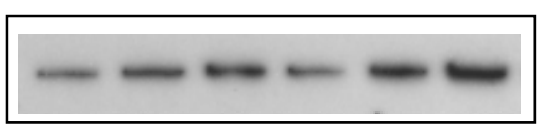

IB : anti-Src
C

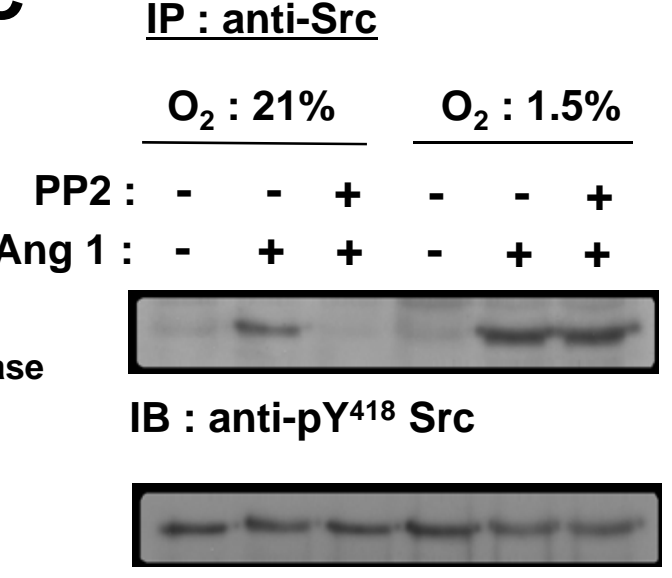

IB : anti-Src
D IP : anti-Tie 2

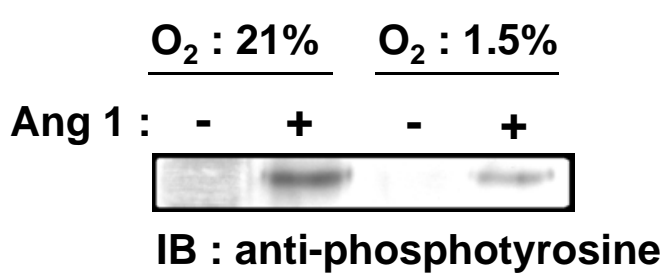

IB : anti-Tie 2

Total extract

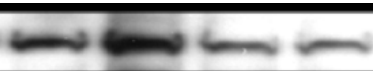

IB : anti- $\beta$-actin

Fig 5. S. Kanda et al. 
A

Hypoxia :
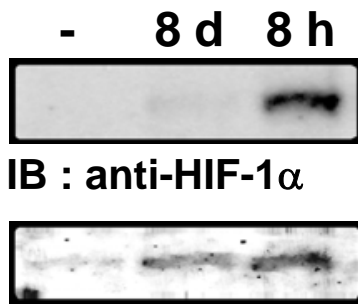

IB : anti-MRP 1

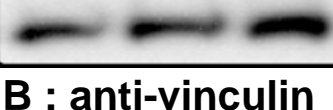

B

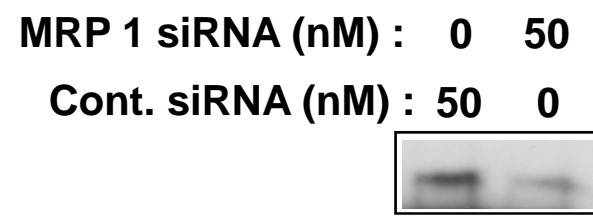

MRP 1/vinculin ratio : $1.00 \quad 0.17$ IB : anti-MRP 1

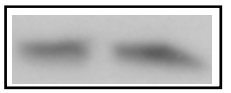

IB : anti-vinculin

Fig. 6. S. Kanda et al.
C

IP : anti-Src

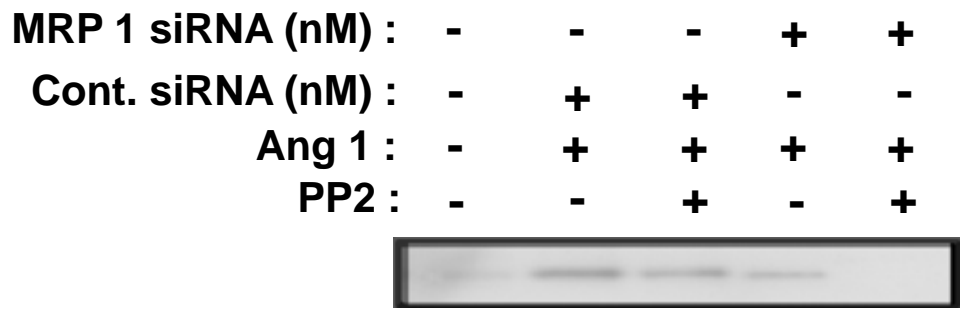

IB : anti-pY ${ }^{418}$ Src

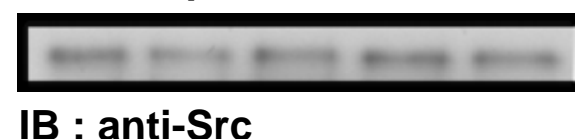

D

Cont. siRNA MRP 1 siRNA

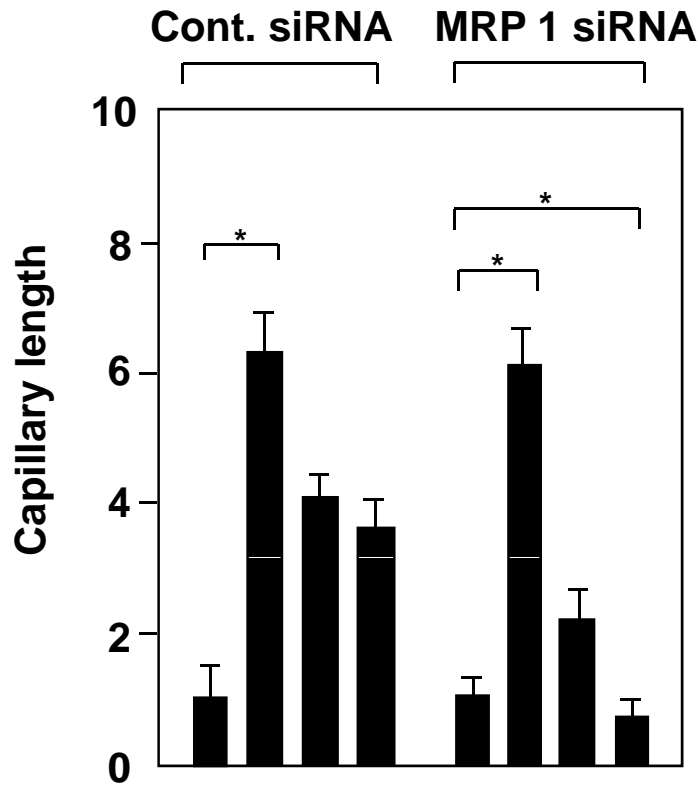

Ang $1+$ PP2 $(1 \mu \mathrm{M})$

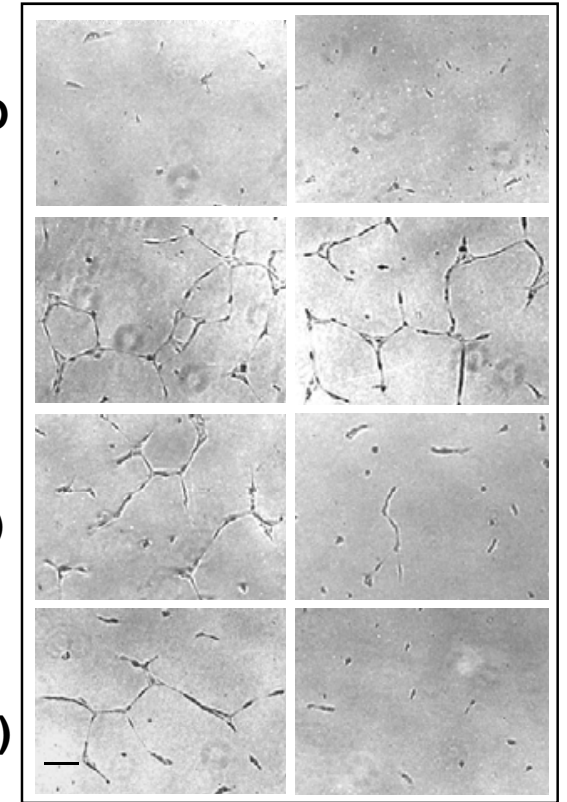

Ang 1: -++++++ PP2 $(\mu \mathrm{M}): \begin{array}{llllllll}0 & 0 & 0.2 & 1 & 0 & 0 & 0.2 & 1\end{array}$ 